\title{
Pós-tratamento de efluente de filtros anaeróbios operados com baixo tempo de detenção hidráulica por escoamento superficial no solo
}

\author{
Post-treatment of anaerobic filters operated with low hydraulic \\ detention time through overland flow system
}

\author{
Adriano Luiz Tonetti \\ Professor Doutor da Faculdade de Engenharia Civil, Arquitetura e Urbanismo da Universidade Estadual de Campinas (Unicamp) - Campinas (SP), Brasil. \\ Bruno Coraucci Filho \\ Professor Titular da Faculdade de Engenharia Civil, Arquitetura e Urbanismo da Unicamp - Campinas (SP), Brasil. \\ Ronaldo Stefanutti \\ Professor Adjunto do Centro de Tecnologia da Universidade Federal do Ceará (UFC) - Fortaleza (CE), Brasil.
}

\begin{abstract}
Resumo
Neste trabalho estudou-se o pós-tratamento de efluente de filtros anaeróbios operados com baixo tempo de detenção hidráulica (TDH) por meio do escoamento superficial no solo. Para isso, foram construídos quatro reatores com volume de $500 \mathrm{~L}$ preenchidos com anéis de bambu e uma rampa com 40,0 m de comprimento e declividade de 3,50\%, coberta com grama Tifton 85 (Cynodon sp). Avaliou-se o tratamento do esgoto doméstico proveniente da cidade de Limeira (SP), operando os filtros anaeróbios com TDH de 3 horas e a aplicação do efluente no escoamento superficial com taxas de 0,10; 0,20; 0,30 e $0,40 \mathrm{~m}^{3} \cdot \mathrm{h}^{-1} \cdot \mathrm{m}^{-1}$. Nestas condições operacionais, o efluente gerado era adequado ao lançamento em corpos hídricos até a aplicação da taxa de $0,20 \mathrm{~m}^{3} \cdot \mathrm{h}^{-1} \cdot \mathrm{m}^{-1}$. Acima desse valor, houve um comprometimento de sua qualidade final em termos de DBO e concentração de fósforo.
\end{abstract}

Palavras-chave: filtro anaeróbio; escoamento superficial; pós-tratamento.

\begin{abstract}
In this investigation the post-treatment of anaerobic filters operated with low hydraulic retention time (HRT) through overland flow system was studied. For that, it was built four reactors whose volume was $500 \mathrm{~L}$, filled up with bamboos rings and a ramp with $40.0 \mathrm{~m}$ of length and inclination of $3.5 \%$, covering with Tifton 85 (Cynodon sp) grass. It was evaluated the wastewater treatment from Limeira city (SP), operating anaerobic filters with 3 hour HRT and the effluent application in superficial drain of $0.10 ; 0.20 ; 0.30$ e $0.40 \mathrm{~m}^{3} \cdot \mathrm{h}^{-1} \cdot \mathrm{m}^{-1}$ rates. In these operational conditions, the effluent was suitable to be discharged into receiving waters until the rate application of $0.20 \mathrm{~m}^{3} \cdot \mathrm{h}^{-1} \cdot \mathrm{m}^{-1}$. Over this value, there was a worse final quality in DBO and phosphorus concentration
\end{abstract}

Keywords: anaerobic filter; superficial drain; post-treatment.

\section{Introdução}

O tratamento de efluentes com o emprego de filtros anaeróbios tem demonstrado grande potencialidade na depuração de esgotos sanitários devido a sua facilidade construtiva e operacional e por apresentar uma significativa remoção de matéria orgânica. Cabe destacar que o Programa de Pesquisas em Saneamento Básico (PROSAB) possibilitou o estudo de materiais alternativos aos tradicionalmente empregados no preenchimento desse tipo de reator, sendo avaliados e aplicados em escala real anéis de eletroduto cortado, escória de alto-forno, anéis de bambu, cascas de coco verde e tijolos cerâmicos (CAMARGO; NOUR, 2001; TONETTI et al., 2010; COUTO E FIGUEIREDO, 1992). No entanto, apesar destes aspectos positivos, esse tipo de reator requer um pós-tratamento para atender os padrões legais de lançamento em corpos hídricos 
Uma possibilidade para complementar o tratamento seria sua associação com o escoamento superficial no solo, um método que tem demonstrado boa eficiência na depuração de águas residuárias, com custos de implantação, operação e manutenção acessíveis a pequenos municípios e indústrias (TAEBI; DROSTE, 2008; SURAMPALLI et al., 1996; CORAUCCI FILHO et al., 2000). A despeito do grande potencial, o escoamento superficial tem sido pouco utilizado no Brasil, além de não existirem muitos estudos que o avaliem no pós-tratamento de reatores anaeróbios (CHERNICHARO et al., 2001). Uma possível explicação para esta conduta pode ser a baixa difusão desta tecnologia no meio especializado, tornando-se de grande importância o aumento da disseminação do conhecimento sobre sua aplicação, contribuindo para a melhoria do saneamento em nosso país (TONETTI et al., 2010).

Seu funcionamento consiste na disposição do efluente líquido na parte superior de terrenos planos construídos que tenham uma pequena declividade e baixa permeabilidade (SURAMPALLI et al., 1996). O efluente percorre por gravidade todo o declive, que é recoberto por uma vegetação (Figura 1). A maior parte do fluxo é coletada na base do terreno, sendo que a percolação é insignificante, pois esse sistema é concebido para solos com baixa permeabilidade (ZIRSCHKY et al., 1989; SURAMPALLI et al., 1996; CHERNICHARO, 2007). A depuração dá-se à medida que o efluente escoa na área recoberta pela vegetação, onde os sólidos em suspensão são filtrados e a matéria orgânica é oxidada pelos microrganismos que se estabelecem nas plantas e no solo.

No tocante ao aspecto operacional, para Tonetti et al. (2010), a utilização de taxas de aplicação muito baixas, em torno de $0,10 \mathrm{~m}^{3} \cdot \mathrm{h}^{-1} \mathrm{~m}^{-1}$, ocasiona a formação de caminhos preferenciais devido à diferença de velocidade entre as linhas de escoamento e pela ausência total de vazão em alguns trechos. Por outro lado, segundo esses mesmos autores, quando o local de instalação do projeto apresenta temperatura e insolação elevadas, podem-se empregar altas taxas, normalmente superiores a $0,30 \mathrm{~m}^{3} \mathrm{~h}^{-1} \cdot \mathrm{m}^{-1}$. Como exemplo de aplicação, na cidade de Itabira (MG), em projeto desenvolvido por Araújo (1998), foram adotadas taxas que oscilaram entre 0,20 a $0,60 \mathrm{~m}^{3} \cdot \mathrm{h}^{-1} \cdot \mathrm{m}^{-1}$. No caso, o efluente final apresentou resultados de demanda bioquímica de oxigênio (DBO) inferiores a $51 \mathrm{mg} . \mathrm{L}^{-1}$ e de sólidos suspensos totais abaixo de 19 mg.L.-1, adequando-o ao lançamento em um corpo hídrico - Decreto no 8.468/1976 (SÃO PAULO, 1976). De forma geral, segundo Surampalli et al. (1996), Zirschky et al.(1989) e Tyrrel, Leeds-Harrison; Harrison (2002), a eficiência desse sistema de tratamento oscila nas proximidades de 90\% para a remoção de $\mathrm{DBO}$ e sólidos suspensos totais.

No que se refere aos nutrientes, o principal mecanismo da remoção de nitrogênio no escoamento superficial ocorre devido à nitrificação seguida de absorção pela vegetação, porém também há a desnitrificação e a volatilização da amônia (ARAÚJO, 1998). Tal característica leva a valores de nitrogênio total Kjeldahl (NTK) decrescentes ao longo do percurso do líquido sobre a rampa, tal como o encontrado por Coraucci Filho et al. (2000). Segundo Wen et al. (2007), a ação sobre o fósforo envolve diversos mecanismos como adsorção, precipitação química, imobilização na forma de compostos orgânicos e remoção pela vegetação. Nesse caso, tem-se que o fósforo passa a ser utilizado no metabolismo da planta durante seu crescimento, indicando que a remoção desse nutriente está ligada à retirada da massa vegetal do sistema de tratamento.

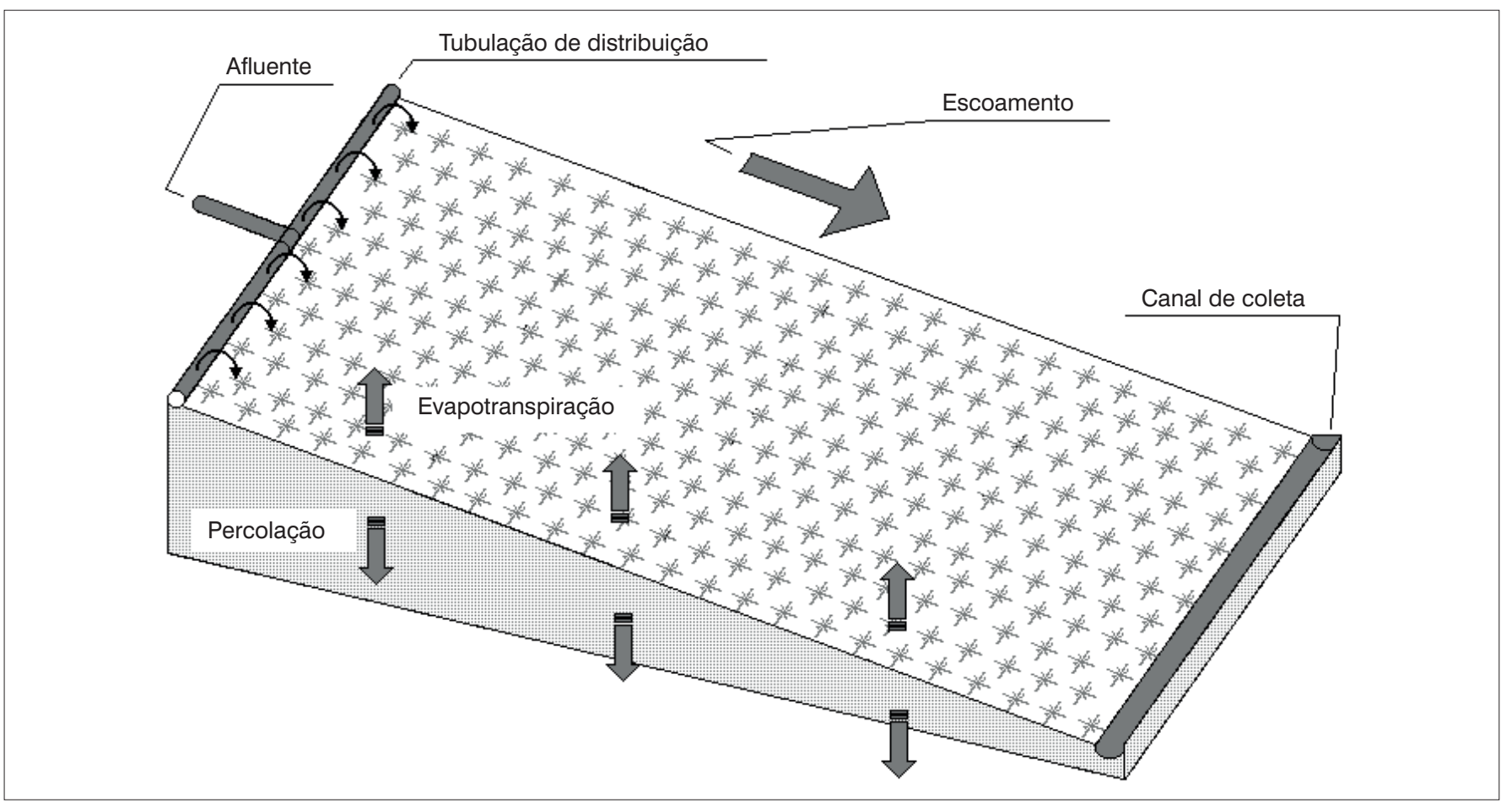

Figura 1 - Esquema de uma rampa de escoamento superficial no solo. 
Baseado nessas informações, na presente pesquisa buscou-se estudar a associação de filtros anaeróbios com o escoamento superficial no solo, sendo que o primeiro reator foi operado com tempo de detenção hidráulica de apenas três horas, valor muito inferior ao recomendado pela norma brasileira (NBR 7.229/1993). Por sua vez, no pós-tratamento com o escoamento superficial, avaliaram-se taxas de aplicação de efluente de 0,10; 0,20; 0,30 e 0,40 $\mathrm{m}^{3} \cdot \mathrm{h}^{-1} \cdot \mathrm{m}^{-1}$, tendo em vista estudar o comportamento desse sistema de tratamento no tocante a remoção de matéria orgânica e nutrientes.

\section{Metodologia}

O projeto foi instalado na área de uma Estação de Tratamento de Esgotos da cidade de Limeira (SP). A água residuária era originária de um conjunto de bairros localizados na periferia do município e, ao chegar à estação, passava pelo gradeamento e caixa de areia. Posteriormente, tinha uma parcela de seu fluxo bombeada a uma caixa de circulação de $1.000 \mathrm{~L}$, localizada a 4,00 m de altura, mantida sempre cheia e com constante circulação de esgoto para a manutenção de uma vazão constante nos filtros anaeróbios.

Pela ação da gravidade, a partir da caixa de circulação, o fluxo de esgoto era direcionado a quatro filtros anaeróbios. Cada um deles construído em aço inox com formato cilíndrico e volume total de 500 L. O fundo era cônico e separado da região ocupada pelo meio suporte por uma grade de bambu, funcionando como um compartimento para a distribuição do esgoto. Na Figura 2 está mostrado um esquema e fotos de um destes reatores.

O meio suporte era constituído de anéis de bambu da espécie Bambusa tuldoides, tendo diâmetro de 0,03 m e cortados em tamanho de aproximadamente 0,05 m de comprimento. Após o preen-

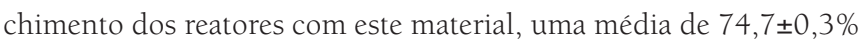
do volume interno era de vazios e a área superficial chegava a $91,8 \pm 1,5 \mathrm{~m}^{2} \cdot \mathrm{m}^{-3}$.
A operação era de fluxo ascendente, com tempo de detenção hidráulica (TDH) nominal de apenas três horas, sendo sua vazão controlada três vezes ao dia. Esse pequeno TDH deveu-se ao fato de que na área de desenvolvimento da pesquisa havia o estudo de diferentes tipos de pós-tratamento para estes reatores, demandando-se uma vazão que não poderia ser atingida empregando-se os valores sugeridos na norma brasileira (NBR 7.229/1993).Assim, adequando-se a tal situação, diminuiu-se o tempo de detenção hidráulica para três horas e buscou-se avaliar o comportamento dos sistemas de pós-tratamento sob esta condição operacional, a qual pode ocorrer momentaneamente quando esta associação de reatores for instalada em escala real em um município ou em uma indústria.

\section{Pós-tratamento por escoamento superficial no solo}

A rampa empregada no escoamento superficial foi construída com uma declividade média de 3,50\%, largura de 4,35 m e 40,00 m de comprimento (Figura 3A). O sistema de distribuição do efluente foi através de um tubo de PVC com diâmetro de $100 \mathrm{~mm}$, possuindo perfurações de $10 \mathrm{~mm}$ a cada $120 \mathrm{~mm}$, garantindo a distribuição do líquido ao longo do terreno.

O efluente dos filtros anaeróbios teve sua aplicação no escoamento superficial avaliada para quatro taxas: 0,$10 ; 0,20 ; 0,30$ e $0,40 \mathrm{~m}^{3} \cdot \mathrm{h}^{-1} \cdot \mathrm{m}^{-1}$, sendo que cada uma delas foi mantida por um período de 14 semanas. A disposição de efluente ocorria diariamente por um período de oito horas, das 8 h00 às 16h00, entre as segundas e as sextas-feiras. A vazão era regulada três vezes ao longo do dia, por meio de válvula instalada na entrada da tubulação de distribuição.

Em intervalos de $5 \mathrm{~m}$ ao longo do declive, foram instaladas barreiras de concreto transversalmente ao comprimento da rampa, com altura de 0,02 m, seguindo a orientação de Paganini (1997). Essas barreiras ou anteparos, apresentados na Figura 3B, visavam regularizar o fluxo do escoamento na rampa durante

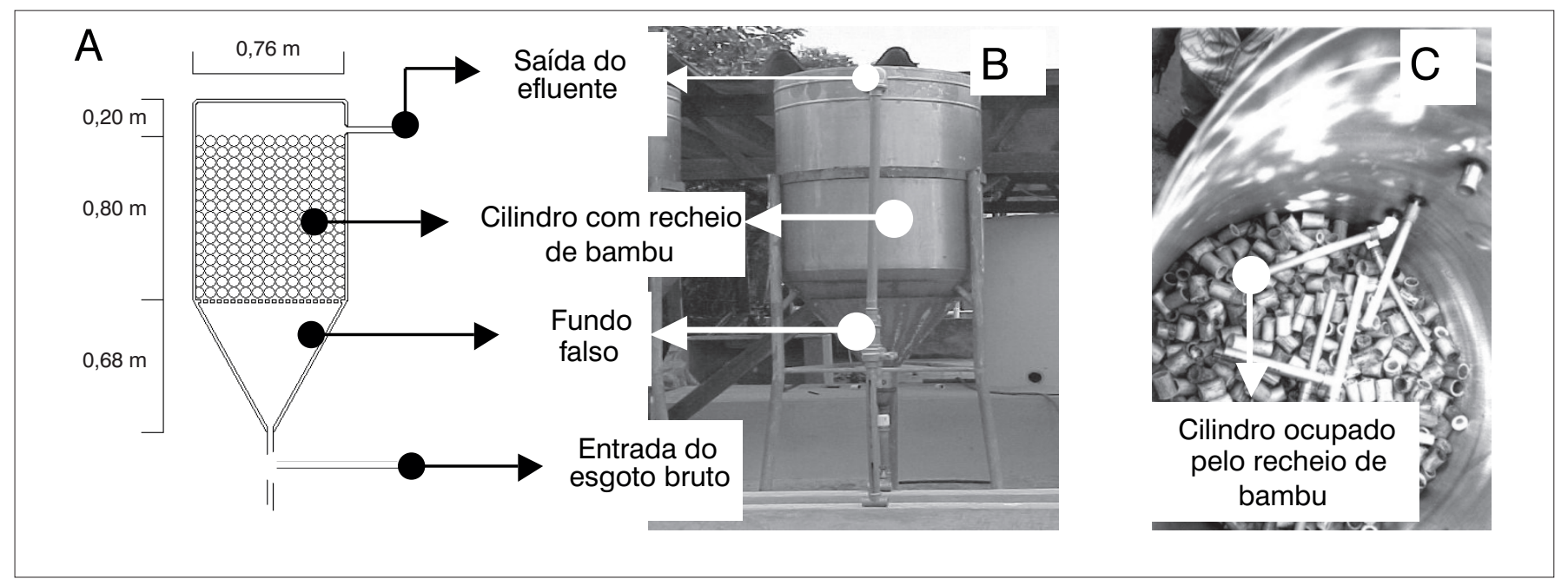

Figura 2 - Esquema de um filtro anaeróbio (A) e sua vista externa $(B)$ e interna $(C)$. 


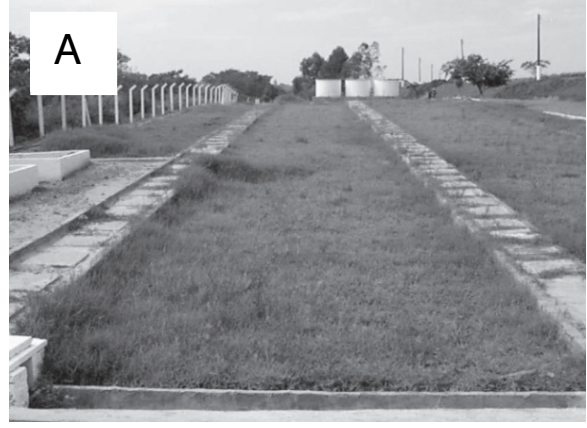

B

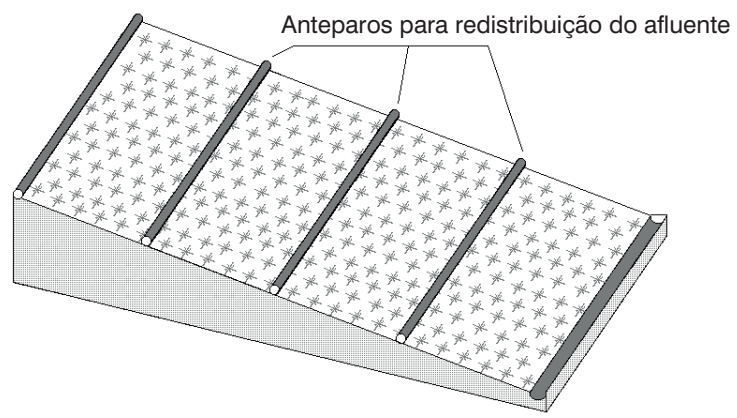

Figura 3 - Sistema de escoamento superficial: vista (A) e esquema (B).

o emprego de baixas taxas de aplicação. A vegetação de cobertura utilizada foi a gramínea Tifton 85 (Cynodon spp) por ter como característica resistir a solos encharcados, ao frio e ao calor (CORAUCCI FILHO et al., 2000).

\section{Coleta das amostras}

As amostras do efluente dos filtros anaeróbios eram obtidas imediatamente após a saída dos quatro reatores, com composição igualitária de cada um deles. No sistema de escoamento superficial, as amostras foram coletadas em uma canaleta instalada na base da rampa. Todas eram obtidas semanalmente, sendo compostas e constituídas por alíquotas obtidas a cada hora e meia, totalizando cinco coletas diárias, que compunham um volume total de $2.000 \mathrm{~mL}$.

As análises de $\mathrm{pH}, \mathrm{DBO}$, demanda química de oxigênio (DQO), NTK acrescido do nitrito e nitrato, e fósforo foram realizadas seguindo as recomendações apresentadas por APHA; AWWA; WEF (2005). Os resultados obtidos foram submetidos a um tratamento estatístico utilizando-se o Programa Bioestat 5.0. Os dados foram analisados quanto à variância e as médias comparadas por meio do teste de Kruskal-Wallis ao nível de 5\% (ps0,05).

\section{Resultados}

A temperatura durante o período de execução da pesquisa foi de $24,5 \pm 5,4^{\circ} \mathrm{C}$ e, por meio da análise da Tabela 1 , nota-se que a DQO média do esgoto bruto, em cada um dos períodos de avaliação das taxas empregadas no escoamento superficial, estava acima da faixa considerada como típica por von Sperling (2005), o qual estipula o mínimo de $400 \mathrm{mg} \mathrm{O}_{2} \mathrm{~L}^{-1}$ e o máximo de $800 \mathrm{mg} \mathrm{O}_{2} \mathrm{~L}^{-1}$ como sendo apropriadas para esgotos de origem doméstica. Este comportamento pode estar relacionado a contribuições clandestinas de efluentes industriais, principalmente relacionada à matéria orgânica não suscetível a degradação biológica, visto que no tocante à $\mathrm{DBO}$, as médias encontradas estavam dentro do limite exposto pelo mesmo autor como característico para esgoto doméstico, o qual situa-se entre 200 e 500 mg. $\mathrm{L}^{-1}$.
Tabela 1 - Resultados obtidos no sistema de tratamento com aplicação das taxas hidráulica de 0,$10 ; 0,20 ; 0,30$ e $0,40 \mathrm{~m}^{3} \cdot \mathrm{h}^{-1} \cdot \mathrm{m}^{-1}$ no escoamento superficial.

\begin{tabular}{|c|c|c|c|}
\hline Parâmetro & Esgoto bruto & $\begin{array}{c}\text { Efluente } \\
\text { dos filtros } \\
\text { anaeróbios }\end{array}$ & $\begin{array}{l}\text { Efluente do } \\
\text { escoamento } \\
\text { superficial }\end{array}$ \\
\hline \multicolumn{4}{|c|}{ Taxa hidráulica de $0,10 \mathrm{~m}^{3} \cdot \mathrm{h}^{-1} \cdot \mathrm{m}^{-1}$ (14 semanas de análises) } \\
\hline $\mathrm{pH}$ & $7,4 \pm 0,4^{\mathrm{a}}$ & $7,2 \pm 0,2^{\mathrm{a}}$ & $7,3 \pm 0,3^{a}$ \\
\hline DBO (mg. $\left.\mathrm{L}^{-1}\right)$ & $355 \pm 83^{a}$ & $166 \pm 70^{\mathrm{b}}$ & $33 \pm 20^{c}$ \\
\hline $\mathrm{DQO}\left(\mathrm{mg} \cdot \mathrm{L}^{-1}\right)$ & $889 \pm 181^{a}$ & $400 \pm 152^{b}$ & $116 \pm 67^{c}$ \\
\hline SST (mg. $\left.\mathrm{L}^{-1}\right)$ & $338 \pm 83^{a}$ & $101 \pm 51^{b}$ & $40 \pm 11^{c}$ \\
\hline Fósforo (mg. $\left.\mathrm{L}^{-1}\right)$ & $5,5 \pm 0,5^{a}$ & $4,5 \pm 0,2^{b}$ & $0,6 \pm 0,4^{c}$ \\
\hline N-total $\left(m g \cdot L^{-1}\right)$ & $48,5 \pm 18,8^{a}$ & $42,4 \pm 15,3^{a}$ & $12,8 \pm 6,1^{b}$ \\
\hline \multicolumn{4}{|c|}{ Taxa hidráulica de $0,20 \mathrm{~m}^{3} \cdot \mathrm{h}^{-1} \cdot \mathrm{m}^{-1}$ (14 semanas de análises) } \\
\hline $\mathrm{pH}$ & $6,9 \pm 0,3^{\mathrm{a}}$ & $7,0 \pm 0,5^{\mathrm{a}}$ & $6,9 \pm 0,4^{\mathrm{a}}$ \\
\hline DBO (mg. $\left.\mathrm{L}^{-1}\right)$ & $364 \pm 201^{\mathrm{a}}$ & $118 \pm 30^{b}$ & $20 \pm 10^{c}$ \\
\hline DQO (mg. $\left.\mathrm{L}^{-1}\right)$ & $923 \pm 298^{a}$ & $389 \pm 162^{\mathrm{b}}$ & $75 \pm 38^{c}$ \\
\hline SST (mg. $\left.\mathrm{L}^{-1}\right)$ & $298 \pm 81^{\mathrm{a}}$ & $121 \pm 23^{b}$ & $24 \pm 23^{c}$ \\
\hline Fósforo (mg. $\left.L^{-1}\right)$ & $8,3 \pm 1,9^{a}$ & $6,9 \pm 0,9^{b}$ & $0,9 \pm 0,5^{c}$ \\
\hline N-total $\left(m g \cdot L^{-1}\right)$ & $52,5 \pm 35,0^{\mathrm{a}}$ & $47,1 \pm 5,8^{\mathrm{a}}$ & $27,6 \pm 11,1^{\mathrm{b}}$ \\
\hline \multicolumn{4}{|c|}{ Taxa hidráulica de $0,30 \mathrm{~m}^{3} \cdot \mathrm{h}^{-1} \cdot \mathrm{m}^{-1}$ (14 semanas de análises) } \\
\hline $\mathrm{pH}$ & $6,8 \pm 0,4^{a}$ & $6,8 \pm 0,3^{\mathrm{a}}$ & $7,0 \pm 0,2^{a}$ \\
\hline $\mathrm{DBO}\left(\mathrm{mg} . \mathrm{L}^{-1}\right)$ & $378 \pm 73^{a}$ & $189 \pm 60^{\mathrm{b}}$ & $67 \pm 19^{c}$ \\
\hline $\mathrm{DQO}\left(\mathrm{mg} \cdot \mathrm{L}^{-1}\right)$ & $969 \pm 192^{\mathrm{a}}$ & $201 \pm 103^{b}$ & $100 \pm 40^{c}$ \\
\hline SST $\left(m g \cdot L^{-1}\right)$ & $280 \pm 91^{a}$ & $126 \pm 72^{\mathrm{b}}$ & $26 \pm 18^{c}$ \\
\hline Fósforo (mg. $\left.\mathrm{L}^{-1}\right)$ & $8,4 \pm 1,5^{\mathrm{a}}$ & $7,6 \pm 0,7^{\mathrm{a}}$ & $2,7 \pm 0,8^{b}$ \\
\hline N-total $\left(m g \cdot L^{-1}\right)$ & $65,9 \pm 21,1^{\mathrm{a}}$ & $64,8 \pm 16,1^{\mathrm{a}}$ & $33,4 \pm 11,2^{b}$ \\
\hline \multicolumn{4}{|c|}{ Taxa hidráulica de $0,40 \mathrm{~m}^{3} \cdot \mathrm{h}^{-1} \cdot \mathrm{m}^{-1}$ (14 semanas de análises) } \\
\hline $\mathrm{pH}$ & $6,9 \pm 0,3^{a}$ & $7,0 \pm 0,4^{\mathrm{a}}$ & $7,2 \pm 0,5^{a}$ \\
\hline $\mathrm{DBO}\left(\mathrm{mg} \cdot \mathrm{L}^{-1}\right)$ & $333 \pm 200^{a}$ & $135 \pm 67^{b}$ & $66 \pm 15^{c}$ \\
\hline DQO (mg. $\left.\mathrm{L}^{-1}\right)$ & $1.158 \pm 301^{\mathrm{a}}$ & $581 \pm 151^{b}$ & $234 \pm 45^{c}$ \\
\hline SST (mg. L-1) & $532 \pm 101^{\mathrm{a}}$ & $300 \pm 53^{b}$ & $69 \pm 43^{c}$ \\
\hline Fósforo (mg. $\left.\mathrm{L}^{-1}\right)$ & $9,6 \pm 1,6^{\mathrm{a}}$ & $9,3 \pm 0,6^{\mathrm{a}}$ & $3,3 \pm 0,9^{b}$ \\
\hline N-total (mg.L-1) & $61,5 \pm 27^{a}$ & $51,1 \pm 13,3^{a}$ & $30,8 \pm 9,4^{b}$ \\
\hline
\end{tabular}

DBO: Demanda bioquímica de oxigênio; DQO: demanda química de oxigênio; SST: sólidos suspensos totais; N-total: nitrogênio total.

a, b, cMédias seguidas pela mesma letra na horizontal não diferem entre si (Kruskal-Wallis 5\%).

A DBO encontrada para todo o período analítico no efluente anaeróbio foi de $156 \pm 68 \mathrm{mg} . \mathrm{L}^{-1}$, não possibilitando a satisfação da legislação do estado de São Paulo - Decreto no 8.468/1976 - (SÃO PAULO, 1976) e demonstrando que este tipo de tratamento demanda outra etapa para a adequação do efluente aos padrões legais e ambientais. Comparativamente, Cruz (2009) ao operar um sistema 
semelhante com TDH de nove horas encontrou valores muito inferiores, que chegaram a $77 \pm 50 \mathrm{mg} \cdot \mathrm{L}^{-1}$, enquanto Cirne et al. (2007) obtiveram uma média de $86 \mathrm{mg} \cdot \mathrm{L}^{-1}$.

Aremoção de DBO e DQO esteve entre as médias de 50 e 58\%, podendo ser correlacionadas com o levantamento apresentado por Pinto (1995), o qual encontrou para uma série de pesquisas em que foram utilizados filtros anaeróbios operados com tempos de detenção hidráulica entre seis e oito horas, eficiências médias que oscilaram de 68 a 79\%. Por sua vez, Tonetti et al. (2011) ao avaliarem um conjunto de filtros anaeróbios operados com TDH de nove horas encontraram remoção média de $76 \pm 12 \%$ em relação a DQO e $71 \pm 15 \%$ em termos de DBO. Destaca-se que o fator que levou a menor eficiência dos filtros anaeróbios no presente trabalho pode ter sido o baixo tempo de detenção hidráulica, que no caso foi de apenas 3 horas, muito abaixo das 12 h recomendada pela NBR 7.229 (1993).

No que se refere à ação deste reator sobre os nutrientes (nitrogênio e fósforo), pode-se afirmar que ela foi bastante insipiente, propiciando remoções que não superaram 19\% para o fósforo e 16\% para o NTK. As médias das concentrações desses parâmetros no esgoto bruto e no efluente dos filtros anaeróbios, nos quatro períodos avaliados, não foram significativamente diferentes (Kruskal-Wallis 5\%), comprovando-se a afirmação de Chernicharo (2007) e de van Haandel e Lettinga (1994) de que os reatores anaeróbios possuem uma capacidade insatisfatória de remover estes compostos.

\section{Avaliação do escoamento superficial no solo}

Ao avaliar-se o pós-tratamento do efluente dos filtros anaeróbios com o escoamento superficial, nota-se por meio da Tabela 1 uma significativa redução dos valores de DBO e DQO. O sistema gerou um efluente adequado ao lançamento em corpos hídricos na aplicação das taxas de 0,10 e $0,20 \mathrm{~m}^{3} \cdot \mathrm{h}^{-1} \cdot \mathrm{m}^{-1}$, visto que os valores médios não ultrapassaram o limite máximo permitido no estado de São Paulo, que é de $60 \mathrm{mg} . \mathrm{L}^{-1}$ em termos de DBO - Decreto no 8.468/ 1976 (SÃO PAULO, 1976). Comparando-se as médias encontradas nestas duas taxas, observa-se também que não houve uma diferença significativa entre ambas (Kruskal-Wallis 5\%), indicando que o uso de uma ou outra não acarretava alteração na qualidade do efluente.

Porém, ao aumentar-se para 0,30 e 0,40 $\mathrm{m}^{3} \cdot \mathrm{h}^{-1} \mathrm{~m}^{-1}$, o efluente apresentou valores médios maiores que o estipulado pela legislação paulista, apontando para um limite da capacidade de tratamento no escoamento superficial nas condições em estudo. Este comprometimento da eficiência do tratamento pode estar associado a um efluente anaeróbio com maior grau de matéria orgânica ainda em sua forma complexa e sem ter sofrido uma hidrólise completa, sendo um indicativo dessa característica o aumento da concentração de sólidos suspensos totais (SST). Como consequência, ao compararmos esses resultados com os obtidos por Paganini (1997), Araújo (1998) e Chernicharo et al. (2001), que empregaram taxas de até $0,60 \mathrm{~m}^{3} \cdot \mathrm{h}^{-1} \cdot \mathrm{m}^{-1}$ e encontraram a DBO do efluente oscilando entre 48 e $52 \mathrm{mg} . \mathrm{L}^{-1}$, nota-se que o sistema em estudo propiciou resultados ligeiramente superiores, atingindo na aplicação de $0,30 \mathrm{~m}^{3} \cdot \mathrm{h}^{-1} \mathrm{~m}^{-1}$ a média de $67 \pm 19 \mathrm{mg} \cdot \mathrm{L}^{-1}$. Por outro lado, Taebi e Droste (2008) ao utilizarem uma taxa de aplicação de $0,35 \mathrm{~m}^{3} \cdot \mathrm{h}^{-1} \cdot \mathrm{m}^{-1}$ no tratamento do efluente de uma lagoa de maturação obtiveram uma DBO média de $77,8 \mathrm{mg}$..-1

Quanto à ação sobre os nutrientes, no caso da concentração de fósforo houve uma redução para $0,6 \pm 0,4 \mathrm{mg} \cdot \mathrm{L}^{-1}$ na taxa de $0,10 \mathrm{~m}^{3} \cdot \mathrm{h}^{-1} \cdot \mathrm{m}^{-1}$ e para $0,9 \pm 0,5 \mathrm{mg} \cdot \mathrm{L}^{-1}$ na de $0,20 \mathrm{~m}^{3} \cdot \mathrm{h}^{-1} \cdot \mathrm{m}^{-1}$. Ambos os dados estiveram abaixo de $1 \mathrm{mg} . \mathrm{L}^{-1}$, valor estipulado no estado do Rio Grande do Sul como o máximo permitido para o lançamento em um corpo hídrico - Resolução CONSEMA n 128/2006 - (RIO GRANDE DO SUL, 2006). Nas taxas de 0,30 e $0,40 \mathrm{~m}^{3} \cdot \mathrm{h}^{-1} \cdot \mathrm{m}^{-1}$, as concentrações médias encontradas estiveram acima desse valor, indicando do mesmo modo que ocorreu para a $\mathrm{DBO}$, que o sistema poderia ter atingido um limite.

No que se refere ao NTK houve uma expressiva remoção após a aplicação do efluente anaeróbio no escoamento superficial, pois os valores em todas as taxas foram significativamente diferentes dos presentes no afluente (Kruskal-Wallis 5\%), demonstrando que o sistema propiciou condições para a sua absorção pela vegetação e para a desnitrificação, além da volatilização da amônia, conforme afirmado por Araújo (1998) e Wen et al. (2007). Comparativamente, Zirschky et al. (1989), ao avaliarem o polimento do efluente de uma estação de tratamento de esgotos, constataram que nas taxas de aplicação de 0,33; 0,43 e $0,57 \mathrm{~m}^{3} \cdot \mathrm{h}^{-1} \cdot \mathrm{m}^{-1}$ em uma rampa de $30 \mathrm{~m}$, os efluentes gerados tinham concentrações de NTK de 17,7, 16,5 e 16,6 mg.L-1 , respectivamente. Tais valores são inferiores aos obtidos na presente pesquisa, conforme pode ser notado pela análise da Tabela 1, porém, cabe destacar que Zirschky et al (1989) empregaram um afluente com uma concentração média de $21,5 \mathrm{mg} \cdot \mathrm{L}^{-1}$ de NTK, ao passo que no presente estudo o efluente anaeróbio apresentou uma média mínima de 42,4 mg. $\mathrm{L}^{-1}$, ocorrida durante a aplicação da taxa de $0,10 \mathrm{~m}^{3} \cdot \mathrm{h}^{-1} \mathrm{~m}^{-1}$. Desse modo, tais resultados apontam para a influência da qualidade do afluente aplicado sobre a concentração de nitrogênio existente no efluente do escoamento superficial.

De forma geral, os dados encontrados nesta pesquisa indicam que para o pós-tratamento de filtros anaeróbios operados com baixo tempo de detenção hidráulica as taxas de aplicação no escoamento superficial não podem superar $0,20 \mathrm{~m}^{3} \cdot \mathrm{h}^{-1} \cdot \mathrm{m}^{-1}$. Ao empregar taxas de 0,30 ou $0,40 \mathrm{~m}^{3} \cdot \mathrm{h}^{-1} \cdot \mathrm{m}^{-1}$ possivelmente haverá a geração de um efluente que não atenda à legislação paulista quando adentre uma vazão afluente que reduza o tempo de detenção hidráulica no filtro anaeróbio. Porém, torna-se importante destacar que será propiciado um amortecimento significativo das oscilações das concentrações de entrada, não levando a um excessivo comprometimento do efluente final, pois a maior média encontrada para a DBO foi de $67 \pm 19 \mathrm{mg} . \mathrm{L}^{-1}$ e de 33,4 $\pm 11,2$ para o NTK. 


\section{Conclusões}

$\mathrm{Na}$ presente pesquisa, constatou-se que um sistema de tratamento de esgotos constituído por filtros anaeróbios operados com tempo de detenção hidráulica de três horas, valor inferior ao sugerido pela norma brasileira (NBR 7.229/1993), associados ao escoamento superficial no solo, apresenta um efluente adequado ao lançamento em corpos hídricos (Decreto no 8.468/1976) até a aplicação da taxa de $0,20 \mathrm{~m}^{3} \cdot \mathrm{h}^{-1} \cdot \mathrm{m}^{-1}$. Acima deste valor de disposição passa a haver seu comprometimento em termos de DBO e concentração de fósforo.

\section{Referências}

APHA; AWWA; WEF. Standard methods for the examination of water and wastewater. (2005) $19^{\text {th }}$ ed. New York: American Public Health Association. $1268 \mathrm{p}$

ARAÚJO, G.C. (1998) Avaliação do pós-tratamento de efluentes de reatores UASB através de um sistema de aplicação superficial de esgotos. Dissertação (Mestrado). Belo Horizonte: Universidade Federal de Minas Gerais. 213 p.

CAMARGO, S.A.R.; NOUR, E.A.A. (2001) Bamboo as ananaerobic medium: effect of filter column height. Water Science and Technology, v. 44, n. 4. p. 63-70.

CHERNICHARO, C.; COTA, R.S.; ZERBINI, A.M.; SPERLING, M.; BRITO, L.H. (2001) Post-treatment of anaerobic effluents in an overland flow system. Water Science and Technology, v. 44, n.4. p. 229-36.

CHERNICHARO, C. (2007) Reatores Anaeróbios. Belo Horizonte: UFMG. $379 \mathrm{p}$.

CIRNE, A.R.C.; ANDRADE NETO, C.O.; MELO, H.N.S.; ARAÚJO, A.L.C. (2007) Avaliação comparativa de filtros anaeróbios contendo diferentes materiais de enchimento. In: $24^{\circ}$ Congresso Brasileiro de Engenharia Sanitária e Ambiental.

RIO GRANDE DO SUL (Estado). Conselho Estadual do Meio Ambiente - CONSEMA. Resolução no 128 de 24 de novembro de 2006. Dispõe sobre a fixação de padrões de emissão de efluentes líquidos para fontes de emissão que lancem seus efluentes em águas superficiais no Estado do Rio Grande do Sul.

CORAUCCI FILHO, B.; NOUR, E.A.A.; FIGUEIREDO, R.F.; STEFANUTTI, R.; KLÜSENER FILHO, L.C.; BROLEZE, S.T. (2000) Estudo do póstratamento de efluente com aplicação do método do escoamento superficial no solo: polimento de efluentes de filtros anaeróbios. In: CHERNICHARO, C.A.L. (coord). Coletânea de trabalhos técnicos. v. 1. Belo Horizonte: ABES. 219 p.

COUTO, L.C.C.; FIGUEIREDO, R.F. (1992) Filtro anaeróbio com bambu para tratamento de esgotos domésticos. In: Congreso Interamericano de Ingenieria Sanitaria y Ambiental. Cuba: La Habana. p. 329-40.

CRUZ, L.M.O. (2009) Tratamento de esgoto sanitário em reator anaeróbio preenchido por casca de coco verde (Cocos nucifera) combinado com filtro de areia. Dissertação (Mestrado). Campinas: Universidade Estadual de Campinas. 163 p.

SÃO PAULO (Estado). Decreto no 8.468 de 08 de setembro 1976. Aprova o regulamento da Lei $n^{\circ}$ 997, de 31 de maio de 1976, que dispõe sobre a prevenção e o controle da poluição do meio ambiente.
ASSOCIAÇÃO BRASILEIRA DE NORMAS E TÉCNICAS - ABNT. NBR 7.229. (1993) Projeto, construção e operação de tanques sépticos. São Paulo: ABNT.

PAGANINI, W.S. (1997) Disposição de esgoto no solo, através de escoamento à superfície, com utilização de gramíneas: avaliação do processo quanto aos aspectos sanitários, operacionais, construtivos e de manutenção. Dissertação (Mestrado). São Paulo: Universidade de São Paulo, 197 p.

PINTO, J.D.S. (1995) Tratamento de esgotos sanitários em filtros anaeróbios utilizando escória de alto forno como meio suporte. Dissertação (Mestrado). Belo Horizonte: Universidade Federal de Minas Gerais. 180 p.

SURAMPALLI, R.Y.; FELLOW, P.E.; CHOU, S.C.; BANERJI, S.K. (1996) Performance evaluation of overland flow wastewater treatment systemunder winter and summer conditions. Journal of Cold Regions Engineering, v. 10, p. 163-77.

TAEBI, A.; DROSTE, R.L. (2008) Performance of an overland flow system for advanced treatment of wastewater plant effluent. Journal of Environmental Management, v. 88, p.688-96.

TONETTI, A.L.; CORAUCCI FILHO, B.; GUIMARÃES, J.R.; CRUZ, L.M.O.; NAKAMURA, M.S. (2011) Avaliação da partida e operação de filtros anaeróbios tendo bambu como material de recheio. Revista Engenharia Sanitária e Ambiental, v. 16, p. 11-6.

TONETTI, A.L.; CORAUCCI FILHO, B.; BERTONCINI, E.I.; OLIVEIRA, R.A.; STEFANUTTI, R. (2010) Avaliação de um sistema simplificado de tratamento de esgotos visando a utilização em áreas rurais. Revista Brasileira de Engenharia Agrícola e Ambiental, v. 14, n. 2, p. 227-34.

TYRREL, S.F., LEEDS-HARRISON, P.B., HARRISON, K.S. (2002) Removal of ammonical nitrogen from landfill leachate by irrigation onto vegetated treatment plane. Water Research, v. 36, n.1, p. 291-9.

VAN HAANDEL, AC.; LETTINGA, G. (1994) Tratamento anaeróbio de esgotos: um manual para regiões de clima quente. Campina Grande: UFPA.

VON SPERLING, M. (2005) Princípios básicos do tratamento de esgotos. Belo Horizonte: UFMG. $452 \mathrm{p}$.

WEN, C.G.; CHEN, T.H.; HSU, F.H.; LU, C.H.; LIN, J.B.; CHANG, C.H.; CHANG, S.P.; LEE, C.S. (2007) A high loading overland flow system: Impacts on soil, characteristics, grass constituents, yields and nutrient removal. Chemosphere, v. 67, p. 1588-600.

ZIRSCHKY, J.; CRAWFORD, D.; NORTON, L.; RICHARDS, S; DEEMER, S. (1989) Ammonia removal using overland flow. Journal of Water Pollution Control Federation, v. 61, n. 7, p. 1225-32. 